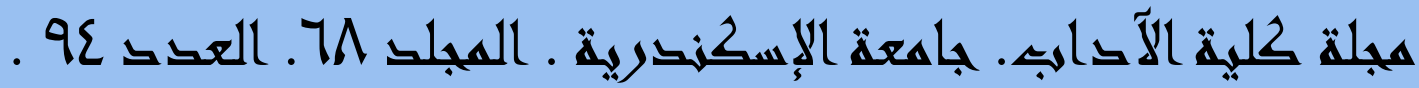

\title{
Eldorado, rêve ou réalité ?
}

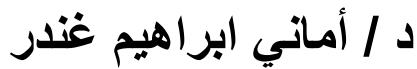 \\ مدرس بقسم اللغة الفرنسية \\ بكلية الآداب - جامعة الاسكندرية
}




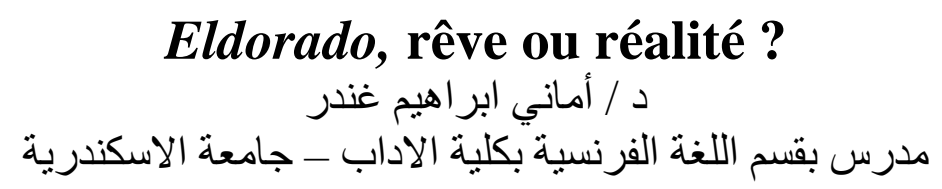

Né à Paris en 1972, Laurent Gaudé est surtout connu pour son œuvre romanesque. Cependant, ce n'est point au roman mais au théâtre que Gaudé a commencé sa carrière d'écrivain. Il étudie les lettres modernes à l'université de Paris III puis passe sa maîtrise qu'il clôture en 1998 par un mémoire intitulé Le Conflit dans le théâtre contemporain.

Ainsi, ses premiers textes sont-ils des pièces de théâtre. Onysos le furieux publié en 1997, a été mis en scène pour la première fois en 2000 au théâtre National de Strasbourg. Il publie également d'autres pièces dont nous pouvons citer à titre d'exemple: Pluie de Cendres (1998), Combat de possédés (1999), Cendres sur les mains (2001) ou encore Maudits les Innocents (2014).

Cependant, très vite, Gaudé se lance dans l'écriture romanesque. Son premier roman Cris paraît en 2001. L'année suivante, La Mort du roi Tsongor lui vaut d'être en lice pour le prix Goncourt mais il ne l'obtient pas. Il remporte cependant le prix Goncourt des lycéens et le prix des librairies. Deux ans plus tard, en 2004, c'est Le Soleil de Scorta qui consacre le vrai succès de Gaudé avec le prix Goncourt ainsi que le prix du jury Jean Giono. Traduit en plusieurs langues avec plus de 80000 exemplaires, ce roman est un véritable succès de librairie. Depuis, ses romans fascinent à la fois le public et la critique. Suivent alors Eldorado (2006), La Porte des enfers (2008), Ouragan (2010), Pour seul cortège (2012) et Danser avec les ombres (2015).

$\mathrm{Si}$ on considère l'inspiration de la totalité de l'œuvre romanesque de Gaudé, on trouve qu'elle se distingue en deux veines essentielles. Certains romans s'inspirent de la mythologie comme c'est le cas pour La Mort du roi Tsongor. D'autres s'inspirent volontiers des événements contemporains et portent directement sur des questions d'actualité comme c'est le cas d'Ouragan (2010) qui aborde l'ouragan Katrina et les dégâts qu'il a causés en ravageant la Louisiane en 2005 ou Danser avec les ombres (2015) où Gaudé évoque le tremblement de terre survenu à Haïti en 2010. 
Or, de tous les romans de Gaudé, nous avons choisi d'étudier Eldorado pour plusieurs raisons. D'une part, ce roman semble lier les deux sources d'inspiration essentielles dans l'œuvre de Gaudé. En se basant sur un mythe, celui de l'Eldorado, Gaudé aborde un des problèmes les plus brûlants de l'actualité, celui de l'immigration clandestine. La mythologie et l'actualité se rejoignent donc dans un roman particulièrement intense aussi bien au niveau thématique qu'au niveau littéraire à travers un sujet dont la complexité et la gravité nous touche tous particulièrement dans les pays méditerranéens.

$\mathrm{Vu}$ le manque d'études sur l'œuvre de Gaudé, et pour aborder Eldorado, nous nous sommes basés dans un premier temps sur les entretiens faits avec l'auteur lors de la publication du roman, autrement dit sur l'épitexte de l'ouvrage pour reprendre la terminologie de Genette. Au cours de ces entretiens, Gaudé laisse l'essentiel de sa pensée. Il explique sa problématique et surtout le sens qu'il veut donner à son roman. Dans un deuxième temps, nous avons choisi d'aborder le roman à travers une analyse structurale et narrative pour essayer de repérer comment Gaudé a pu traiter le problème de la migration clandestine d'un point de vue littéraire.

En effet, Eldorado traite d'une des questions les plus brûlantes qui secouent la région : l'immigration clandestine. Ce sujet assez grave dont on entend parler quotidiennement dans les médias, sans pour autant pouvoir démêler le vrai du faux, le fascine. Toutefois, dans les journaux, Gaudé refuse ces «mêmes mots, le même format, journalistique, court et formel. Ce n'est pas une critique, c'était le rôle des médias de traiter ce sujet de cette façon. Mais je me suis dit que ce thème était tellement important qu'il méritait un temps plus long et un ton plus empathique.»"

Il décide donc d'aborder ce sujet hautement actuel par le biais de la littérature. C'est ainsi qu'il a commencé à creuser et lire sur le sujet en découpant des articles de journaux. Il a trouvé passionnant un sujet au carrefour de multiples thèmes : l'identité, le sentiment d'appartenance, les

\footnotetext{
1 «Entretien avec Laurent Gaudé, écrivain engagé », $1^{\mathrm{er}}$ octobre 2014, Propos recueillis par Virginie PLAUT, https://blogs.mediapart.fr/amnesty-international/blog/011014/entretien-avec-laurent-gaudeecrivain-engage, consulté le 12 mai 2018.
} 
voyages, les rencontres, l'attachement à un pays, le désir, la violence, la déchirure...

«J'ai des dossiers dans lesquels je conserve des articles que je découpe dans les journaux. Quand je me suis mis à travailler sur ce qui allait devenir Eldorado, j'ai ressorti le dossier émigration. Il contenait des articles de 1999-2000, que j'avais un peu oubliés, même si l'idée me trottait dans la tête. Il y a eu des éléments déclencheurs tels que l'histoire des bateaux affrétés du Liban par les services secrets syriens pour mettre pression sur l'Europe. Cela m'avait frappé parce que je découvrais un peu nä̈vement derrière tout ça des questions de géopolitique et de diplomatie indirecte. L'autre élément déclencheur fut d'apprendre que pour la mafia des Pouilles, en Italie, l'argent généré par le trafic d'immigrés est devenu supérieur à l'argent généré par le trafic de drogue. Ces chiffres m'ont beaucoup marqué. » ${ }^{2}$

Il est vrai que, liés au problème de la mondialisation, aux conflits, à la pauvreté et à l'inégalité des revenus, les flux migratoires n'ont cessé de s'amplifier au cours des dernières années. La question de l'immigration est effectivement au cœur de l'actualité et des débats actuels. Depuis les événements qui ont secoué les pays du Proche et Moyen Orient, les guerres qui ont frappé la région: la guerre en Syrie depuis 2011 et jusqu'à aujourd'hui, l'intervention militaire en Libye puis la guerre civile, les soulèvements en Tunisie, la guerre en Somalie ou la dictature en Érythrée, ils sont toujours de plus en plus nombreux ceux qui décident de quitter leur pays pour fuir la pauvreté et l'instabilité et se lancer sur la route de l'Europe à la recherche d'une vie meilleure.

Force est de constater que depuis la publication en 2006 de Eldorado, la question n'a pas cessé d'être d'actualité. Entre 2000 et 2010, le nombre total de migrants a doublé par rapport à la décennie précédente. Ce nombre augmentait de 2 millions par an par rapport aux années quatre-vingt-dix. Ce chiffre est passé à 4,6 millions par an à partir des années deux mille.

\footnotetext{
2 «Interview de Laurent Gaudé. Des histoires et des hommes », Propos recueillis par Thomas FLAMERION, pour Evene.fr, Août 2006, http://evene.lefigaro.fr/livres/actualite/laurent-gaude-intervieweldorado-soleil-scorta-430.php, consulté le 13 mars 2018.
} 
L'agence Frontex, chargée des frontières extérieures de l'espace Schengen, estime 340000 le nombre de personnes qui ont tenté de rejoindre l'Europe sur les sept derniers mois de 2015, contre 123000 en 2014, la majeure partie des migrations provenant de l'Afrique subsaharienne et d'Afrique du Nord.

Il va sans dire que cette migration a des conséquences assez graves sur les plans politique, social, démographique et culturel, mais aussi et surtout ces migrations soulèvent de nombreuses questions en matière de droits de l'homme et c'est justement cette question que tente d'aborder Gaudé dans son roman.

Aujourd'hui, plus que jamais, chaque jour nous apporte plus de nouvelles macabres à la télé ou dans les journaux : des personnes asphyxiées dans des camions, des personnes noyées ou disparues au large... Qu'il soit terrestre ou maritime, le parcours des immigrants est extrêmement dangereux et se déroule dans des conditions déplorables. Ils voyagent entassés dans des camions ou dans des bateaux surchargés souvent abandonnés en plein mer par les passeurs. Ces traversées sont également très coûteuses.

Leurs voyages semble-t-il absurdes ? leurs rêves dérisoires voire inexistants ? Ceci est peut-être vrai mais Gaudé nous rappelle que tant que la misère régnera en Afrique, impossible d'empêcher ces hommes de poursuivre leur rêve de rejoindre l'Europe pour un avenir meilleur.

Le vrai apport du roman de Gaudé est bien de pouvoir proposer un autre point de vue sur la question de l'immigration clandestine, un point de vue loin de la politique, loin des rapports de média, un point de vue essentiellement humain. Le roman est beaucoup plus que le récit de traversée d'un migrant. Il expose les deux points de vue : celui d'un européen et celui d'un migrant et ceci à travers une structure très élaborée.

En effet, au niveau littéraire, la structure du roman est particulièrement travaillée. Laurent Gaudé a d'ailleurs confié avoir beaucoup travaillé pour donner au roman cette dynamique particulière ${ }^{3}$. Tout le roman est placé sous le signe de la dualité et se trouve marqué par un rythme binaire très prononcé. Au niveau structural, et malgré le nombre impair des chapitres, le roman propose deux histoires complètement différentes, deux

\footnotetext{
${ }^{3}$ Émission «Des mots de minuit », 8 novembre 2006, France 2, Présentée par Philipe LEFAIT, http://www.ina.fr/video/I13189820/laurent-gaude-eldorado-video.html, consulté le 12 mars 2018.
} 
récits donc, deux voyages et deux quêtes. Or, c'est précisément cette structure qui témoigne de l'essentiel du roman de Gaudé car elle se trouve mise au service de ce que l'écrivain cherche à présenter, un autre point de vue sur la question de l'immigration clandestine. S'il arrive à proposer un point de vue, il excelle en même temps à mettre la lumière sur le point de vue opposé.

Or, pour pouvoir exposer les différents points de vue au sujet des migrants, Gaudé met au centre de son roman deux personnages principaux qui incarnent chacun une facette complètement différente de la migration : un européen et un africain. Si Salvatore Piracci incarne le point de vue européen sur la question de la migration, Soleiman, lui, présente le point de vue de ceux qui partent. Le premier, âgé de 40 ans, est un commandant italien intégré à la marine italienne depuis l'âge de 20 ans, à Bari, dans les Pouilles. A l'âge de 37 ans, il est devenu le commandant de la frégate Zeffiro. Son travail consiste à surveiller la mer au large de l'île Lampedusa, porte d'entrée des migrants venus d'Afrique par la Méditerranée. Quant à Soleiman, c'est un jeune Soudanais âgé de 25 ans qui décide, avec son frère Jamal, de tenter leur chance en Europe.

Les deux personnages reflètent donc les deux points de vue opposées sur la question de l'immigration clandestine. C'est le Nord contre le Sud, l'Europe contre l'Afrique, le commandant qui lutte contre les migrants et le migrant déterminé de poursuivre son rêve pour arriver en Europe. Or, c'est justement l'opposition entre ces deux personnages principaux qui, d'ailleurs, ne se connaissent pas qui permet à Gaudé d'exposer des points de vue différents, voire contradictoires sur la question.

Or, si on regarde de près les deux personnages, on trouve qu'au niveau onomastique, ils sont traités différemment. Alors que nous connaissons, le nom et le prénom du commandant italien : Salvatore Piracci, nous n'avons que le prénom de Soleiman. Notons qu'en italien, Salvatore veut dire «sauveur». Il est vrai que le travail du commandant Piracci consiste à sauver les clandestins qui échouent sur les côtes italiennes. Quant à l'absence de nom pour le jeune Soudanais, il l'explique lui-même. Une fois décidé à partir pour l'Europe, il comprend parfaitement que son nom ne sera plus utilisé : 
«Nous allons laisser derrière nous la tombe de nos ancêtres. Nous allons laisser notre nom, ce beau nom qui fait que nous sommes ici des gens que l'on respecte. [...] Nous laisserons ce nom ici, accroché aux branches des arbres comme un vêtement d'enfant abandonné que personne ne vient réclamer. Là où nous irons, nous ne serons rien. " ${ }^{4}$

Puis à chacun de ces personnages principaux équivaut un récit, complètement différent et indépendant. La dualité des personnages principaux est savamment transposée dans la construction du roman.

En effet, dès le premier abord avec le roman, le lecteur retrouve que le roman comprend treize chapitres bien distincts au niveau typographique. Chacun est numéroté et pourvu d'un titre en rapport avec le contenu du chapitre.

Cependant, très vite, le lecteur se trouve confus. Le roman demande sa participation pour pouvoir reconstruire de chapitre en chapitre le fil narratif de deux récits différents. Bien que la numérotation des chapitres ne laissent prévoir aucune dualité, le roman livre deux récits distincts en alternance. Tandis que les chapitres impairs retracent le destin de Salvatore Piracci, les chapitres pairs abordent l'histoire de Soleiman.

Ainsi, le lecteur plonge-t-il dans deux mondes différents, et ceci dès l'incipit, ou plutôt les deux incipit car chaque récit commence différemment. Les premières pages fournissent l'incipit du récit de Piracci. Elles commencent par une description du port de Catane, un jour de marché. Avec un grand réalisme, les détails visuels et auditifs abondent.

«A Catane, en ce jour, le pavé des ruelles du quartier du Duomo sentait la poiscaille. Sur les étals serrés du marché, des centaines de poissons morts faisaient briller le soleil de midi, des seaux, à terre, recueillaient les entrailles de la mer que les hommes vidaient d'un geste sec. ${ }^{5}$

Cette description sert de toile de fond au texte avant l'apparition de Piracci qui se promène dans ce décor.

\footnotetext{
${ }^{4}$ Eldorado, Actes Sud, 2006, p. 46.

${ }^{5}$ Ibid., p.9.
} 
Le second incipit débute in medias res, au deuxième chapitre, par un monologue intérieur. Soleiman est en train de faire ses adieux à sa ville. Il évoque son départ imminent avec son frère.

«Je suis avec mon frère Jamal. [...] Je sais que nous partirons cette nuit.[...] S'il m'a demandé de venir avec lui, c'est qu'il veut que nous soyons ensemble pour dire adieu à notre ville. Je ne dis rien. La tristesse et la joie se partagent en mon âme. [...] J'ai doucement mal de ce pays que je vais quitter. " ${ }^{6}$

Soleiman nous livre ses pensées et ses sentiments intenses et confus. Sa nostalgie se manifeste déjà. S'il quitte difficilement sa ville, sa maison et sa mère, le départ est nécessaire. Bien qu'il s'inquiète de l'avenir, il est rassuré d'être avec son frère aîné. L'inquiétude se trouve mêlée avec la joie de pouvoir partir. Dans ce premier chapitre du récit de Soleiman, la force des sentiments de fraternité éloigne les sentiments de peur d'où le titre du chapitre «Tant que nous serons deux».

Au niveau de la narration, Eldorado est un texte mêlé dans lequel deux voix se font entendre à tour de rôle, chapitre après chapitre. Cette double narration permet de rompre la monotonie du récit. Pour chacune d'entre elles, l'auteur adopte une focalisation différente. Dès les premières lignes de chacun des deux récits, le lecteur arrive à percevoir le point de vue selon lequel le récit avance.

Ainsi, le récit de Piracci commence-t-il par un tableau du port de Catane. L'auteur adopte une narration à la troisième personne selon une focalisation interne. Les pensées et les impressions de Piracci sont ainsi révélées au lecteur. C'est par son œil que nous percevons les choses.

Par contre, le récit de Soleiman, qui débute par un monologue intérieur, est rapporté à la première personne du singulier. Ce personnagenarrateur dévoile lui-même directement sa propre perception des événements, ainsi que ses sentiments et ses pensées.

Le recours à ces deux points de vue narratifs distincts témoignent d'une part de la dualité des récits. D'autre part, il affronte le lecteur à une multiplicité de points de vue au sens propre comme au sens narratif du

\footnotetext{
${ }^{6}$ Ibid., p.43.
} 
terme. En fait, la réalité n'est pas à chercher dans un seul point de vue, elle est effectivement diverse et c'est justement ce que cherche à montrer Laurent Gaudé.

Le choix de la focalisation renforce également l'analyse des personnages. Salvatore paraît distant, pessimiste et sombre. La $3^{\text {ème }}$ personne renforce ce sentiment d'éloignement. Soleiman, en revanche, est jeune et plein de vie. Le lecteur peut s'identifier à lui et ce n'est qu'en s'identifiant à lui que l'on pourrait vraiment ressentir les douleurs des migrants. L'usage de la $1^{\text {ère }}$ personne favorise cette proximité. Gaudé, en donnant la voix à Soleiman, donne la voix aux migrants, à ceux qui n'ont point de voix dans les journaux et les rapports de média.

Par ailleurs, que ce soit à travers la narration à la $1^{\text {ère }}$ personne ou la focalisation interne à la $3^{\text {ème }}$ personne, le lecteur est capable de suivre les personnages dans leurs pensées et surtout dans leurs transformations. En fait, les deux personnages d'Eldorado ne sont point figés. Ils évoluent au cours de l'histoire et connaissent de profondes transformations psychologiques ce qui nous permet de comparer Eldorado au genre du récit initiatique.

Ce genre de roman, né au $\mathrm{XIX}^{\mathrm{e}}$ siècle, met en scène l'évolution et la transformation qui arrive au héros au fur et à mesure des expériences et des épreuves au bout desquelles il acquiert une certaine maturité. Eldorado, encore une fois, met le lecteur face à deux récits initiatiques qui montrent l'essentiel de la pensée de Gaudé.

$\mathrm{Si}$ on essaye de suivre les deux personnages dans leur voyage initiatique, on trouve qu'au début du roman, le lecteur rencontre Salvatore Piracci, le commandant qui récupère les migrants en mer depuis 20 ans. Il représente 1'autorité et obéit aux ordres sans poser de questions. "Ils nous disaient que nous étions là pour garder les portes de la citadelle. Vous êtes la muraille de l'Europe. C'est ce qu'ils nous disaient. [...] C'est une guerre, messieurs. [...] Ils sont toujours plus nombreux et la forteresse Europe a besoin de vous. $»^{7}$

Très vite, il fait une première rencontre décisive qui le bouleverse : la femme du Vittoria. Cette inconnue qui est venue demander à Piracci son aide est une immigrée qui a vu mourir son enfant de soif lorsque le bateau sur lequel elle avait embarqué du Liban a été abandonné à la dérive par les

\footnotetext{
Ibid., p. 67.
} 
passeurs. Obstinée, elle demande à Salvatore une arme pour venger la mort de son enfant en tuant l'homme d'affaires syrien véreux qui a affrété le bateau. Après avoir essayé en vain de la dissuader et face à son inébranlable volonté, Piracci lui donne son arme. «Parce qu'elle le voulait, [...]. Elle le voulait de tout son être. ${ }^{8}$ Elle était «comme un bloc dur de volonté ${ }^{9}$. La femme disparaît, laissant le commandant vide «D'un vide confortable qui le dégoûtait. ${ }^{10}$

Le «sauveur » sauve donc la femme du Vittoria en l'aidant à réaliser son rêve de vengeance. Mais cette première rencontre le bouleverse profondément. Il envie son courage et sa détermination. Tout d'un coup, le dégoût, la colère et le doute s'emparent de lui. «Je me dis que je ne suis que la malchance, le visage laid de la malchance. [...] depuis près de vingt ans, je promène ma silhouette sur la mer et je suis le mauvais oil qui traque les désespérés. C'est de cela que je suis épuisé. » ${ }^{11}$ En échange de son arme, la femme du Vittoria lui a donné l'insatisfaction. "Le dégoût ne lui laissait guère de répit. [...] Elle lui avait offert cela, peut-être, la gifle des pauvres, l'impérieux besoin de désirer. ${ }^{12}$ On ignore si la femme du Vittoria arrive à ses fins. Cependant, son rôle est accompli. Elle a déclenché la remise en question chez Salvatore de toute sa vie en le forçant à commencer sa recherche identitaire.

Plus tard, à la recherche d'autres barques laissées par les passeurs au large de la mer durant une tempête, Piracci ne cherchait qu'à sauver ces pauvres migrants. "Au fond, ces histoires d'émigration et de frontières n'étaient rien. [...] à cet instant précis, il n'y avait plus de bâtiment de la marine militaire et de mission d'interception. Il n'y avait plus d'Italie et de Libye. [...] Ses hommes partaient sauver d'autres hommes, par une sorte de fraternité sourde. ${ }^{13}$

La foi et la nécessité de sa tâche l'avait définitivement quitté. "C'était cela, oui. Le gardien de la citadelle était fatigué tandis que les assaillants étaient sans cesse plus jeunes. Et ils étaient beaux de cette lumière que

\footnotetext{
Ibid., p. 61.

Ibid, p. 42.

Ibid., Loc.cit.

Ibid., Loc.cit.

Ibid., p. 68.

Ibid., p. 73
} 
donne l'espoir au regard. ${ }^{14}$ Son travail commençait à le dégoûter. Il repoussait des hommes qu'il enviait chaque jour un peu plus.

Une deuxième rencontre décisive advient dans le cimetière de Lampedusa où Piracci s'est trouvé face à des tombes sur lesquelles il y avait des croix en bois mal implantées, datées mais sans noms. Ce sont les tombes des premiers migrants. Un homme apparait comme un fantôme et évoque devant Piracci «le cimetière de l'Eldorado ». C'est justement l'endroit où le terme eldorado est pour la première fois cité dans le roman.

En effet, le roman de Gaudé tire son origine du mythe de l'Eldorado. Le terme eldorado vient de l'espagnol el dorado, qui signifie « le doré ». Au début du $16^{\mathrm{e}}$ siècle, lorsque les conquistadors sont arrivés en Amérique du Sud, le récit d'une tribu indigène située dans les hauteurs des Andes, dans l'actuelle Colombie, leur a été conté. Cette contrée fabuleuse est supposée regorger d'or. Or, comme toute légende survivant des siècles, le mythe de l'Eldorado repose sans doute sur une réalité. Les récits racontent que lors de l'arrivée au pouvoir d'un nouveau chef de tribu, une cérémonie au lac Guatavita inaugure son règne. Si les récits de cette cérémonie diffèrent, tous décrivent le nouveau dirigeant recouvert de poudre d'or et indiquent que de l'or et des bijoux précieux étaient jetés dans le lac comme offrande aux dieux. Les Espagnols ont alors baptisé ce chef El dorado, «le doré». Les conquistadors, ayant entendu parler de cette région, sont partis à la recherche de l'or anéantissant sur leur passage des peuples et des civilisations entières, mais ils n'ont jamais atteint le précieux trésor.

Depuis, ce mythe est devenue très populaire. Il est, en outre, évoqué par Voltaire dans Candide (1759) comme une contrée utopique dont rêve tout Français hostile à la royauté de droit divin. De façon générale, le terme ayant évolué, l'eldorado symbolise un pays de rêves, un monde parfait utopique où règnent prospérité, justice et bonheur.

Fasciné par le terme qu'il emploie dans le titre, Gaudé explique l'ambiguïté du terme Eldorado au cours d'un entretien. Pour lui, c'est un mot fascinant car à partir du moment où on le prononce on sait que c'est faux. «On sait très bien qu'eldorado n'existe pas, pourtant le mot existe.» ${ }^{15}$

14 Ibid., p. 117.

15 Émission «Des mots de minuit». 
C'est un mot concret qui désigne un lieu qui n'existe pas. Il symbolise le rêve et le désir des hommes d'une vie meilleure. Mais il représente en même temps l'inaccessibilité, l'impossibilité d'atteindre le rêve car effectivement tout le monde sait qu'il n'existe pas. L'eldorado est donc le symbole des rêves brisés, inaccessibles.

Pourtant, Gaudé insiste sur le fait que chacun a besoin de ce rêve au fond de lui. "Eldorado est un mot magique, un fantasme qui fonctionne pour chacun d'entre nous, affirme-t-il. [...] C'est cette espèce d'insatisfaction et de désir tendu vers un petit ailleurs, un peu plus loin, un peu mieux. Cette quête, qui nous fait avancer, est en chacun d'entre nous. [...] Le mot contient sa folie. C'est la chimère absolue à laquelle on ne peut s'empêcher de croire. » 16

Or, dans le roman de Gaudé, c'est l'homme-fantôme du cimetière de Lampedusa qui explique à Piracci pourquoi ces milliers de clandestins quittent leurs pays et leurs familles et prennent ce chemin dangereux et macabre pour arriver en Europe. Ils sont en fait à la recherche de l'eldorado :

"L'herbe sera grasse, dit-il, et les arbres chargés de fruits. De l'or coulera au fond des ruisseaux, et des carrières de diamant à ciel ouvert réverbéreront les rayons du soleil. Les forêts frémiront de gibier et les lacs seront poissonneux. Tout sera doux là-bas. Et la vie passera comme une caresse. L'Eldorado, commandant. Ils l'avaient au fond des yeux. Ils l'ont voulu jusqu'à ce que leur embarcation se retourne. En cela, ils ont été plus riches que vous et moi. Nous avons le fond de l'œil sec, nous autres. Et nos vies sont lentes. ${ }^{17}$

Cette rencontre est effectivement décisive et marque profondément le commandant. Ils parvient pour la première fois à comprendre la détermination de ces clandestins, leur volonté et surtout l'espoir qui les anime. Il envie de plus en plus ces migrants, qui ont la fièvre dans les yeux,

\footnotetext{
${ }^{16}$ «Entretien. Laurent Gaudé. Eldorado ou la chimère qui nous fait croire », 25 septembre 2006, Isabelle FALCONNIER, https://www.payot.ch/fr/selections/payot-1\%27hebdo-/septembre-2006-les-meilleursromans-de-la-rentr-eacute-e-/entretien-laurent-gaudé-eldorado-ou-la-chimère-qui-nous-fait-croire, consulté le 20 mai 2018.

${ }^{17}$ Eldorado, p.p. 120, 121.
} 
qui sont riches car ils ont la richesse de ceux qui ne renoncent pas, de ceux qui rêvent toujours plus loin.

Mais cette rencontre permet aussi à Piracci de comprendre aussi que chacun a son propre Eldorado, son rêve. Il découvre que contrairement à ces migrants, lui, est vide. Aucun rêve ne l'anime. C'est ce qui lui donne envie de poursuivre sa route pour atteindre une sérénité qu'il ne connaît pas.

Piracci décide d'abandonner cette vie qui ne lui convient plus. Il décide d'aller à la recherche de son eldorado qui, pour lui, existe au pays de ces migrants. Il décide d'effectuer le trajet inverse. Au bord d'un petit bateau, il quitte Catane et part pour l'Afrique ayant laissé ses papiers d'identité. Piracci veut partager l'expérience des immigrés. En plein mer, dans la nuit noire, il laisse derrière lui l'ombre de Lampedusa, pourtant l'île rêvée de tout immigré.

En entreprenant le voyage dans le sens inverse des clandestins, du nord au sud, il va à la recherche de son eldorado, de ce qu'il a vu dans le regard des migrants et qu'il leur envie : la volonté, l'espoir et la détermination. Il savait bien "qu'il allait au-devant de pays où la terre se craquelle de faim. Mais il y avait l'Eldorado tout de même, et il ne pouvait s'empêcher d'y rêver. La vie qui l'attendait ne lui offrirait ni or ni prospérité. Il le savait. Ce n'est pas cela qu'il cherchait. Il voulait autre chose. Il voulait que ses yeux brillent de cet éclat de volonté qu'il avait souvent lu avec envie dans le regard de ceux qu'il interceptait. » 18

Ainsi, dans le roman de Gaudé, l'eldorado acquiert-il différents sens pour chacun des personnages du roman qui sont tous en quête du pays rêvé, de « leur » Eldorado. Pour les migrants que représente Soleiman, l'Eldorado c'est l'Europe qui, pour eux, représente le seul moyen d'accéder à une vie riche et prospère. Pour Piracci, l'eldorado est plus abstrait. C'est le rêve qu'il veut poursuivre, le chemin qu'il cherche afin d'accéder à une sérénité qu'il ne connaît pas. L'Eldorado est un certain accomplissement qu'il n'arrive pas à atteindre. Le voyage de Piracci est un voyage initiatique à la recherche de soi, c'est une recherche identitaire qu'il entend mener. Il découvre également que l'effort seul fait pour y arriver et la lutte elle-même sont capables de remplir la vie de l'homme.

${ }^{18}$ Ibid., p. 147 
L'autre rencontre décisive est bien à Ghardaïa lorsque Piracci entend un conteur africain parler aux migrants de la légende de Massambalo : le dieu des immigrés qui laisse ses «ombres » sillonner le continent. «Ces ombres ne disent rien. C'est à travers elles que Massambalo voit le monde. [...] Ces ombres sont toujours en route. " ${ }^{19} \mathrm{~S}$ 'il arrive à un migrant de les rencontrer, il doit leur laisser un cadeau et si l'ombre de Massambalo accepte l'offrande, ceci veut dire que le voyage se passera bien.

Face à cette rencontre, un sorte de dégoût submergea Piracci «sans qu'il sût si c'était parce qu'il ne pouvait partager cet enthousiasme ou si c'était de constater qu'une telle crédulité puisse exister. Les deux sentiments se mêlaient en lui. ${ }^{20}{ }^{2}$ Mais il découvre en même temps qu'il ne croit en rien. Il envie leur foi. "Il aurait aimé y croire, lui aussi. Que ses yeux brillent de la même joie aveugle. [...] Mais il était sec. Et usé. Plus rien ne pouvait ranimer son regard. ${ }^{21}$ Il prend conscience que l'eldorado n'est pas pour lui. "'Je ne vis plus pour rien", pensa Salvatore Piracci en s'éloignant du petit groupe d'hommes. [...] Je suis tari comme une vieille outre sèche. Plus rien en moi qui me donne envie. [...] Il est temps de mourir. [...] Son corps pouvait encore durer. Il n'était ni vieux, ni malade. Mais l'esprit était sec. $\gg^{22} \mathrm{Ce}$ moment marque effectivement le début de sa chute.

De l'autre côté, Soleiman mène son propre voyage initiatique. Dans le deuxième chapitre, il arrive, avec son frère Jamal, en Libye. Une première frontière a été franchie. Cette frontière est invisible, aucun changement de paysage entre le pays d'origine et le pays voisin. Les frontières entre le Soudan et la Libye n'ont ni postes frontières ni fils barbelés. Mais c'est à ce moment qu'il apprend que son frère est malade, qu'il ne pourra pas continuer avec lui le trajet et qu'il devra partir seul. «Je me suis trompé. Aucune frontière n'est facile à franchir. Il faut forcément abandonner quelque chose derrière soi. [...] Aucune frontière ne vous laisse passer sereinement. Elles blessent toutes. ${ }^{23}$

Soleiman quitte donc son frère qui lui laisse son collier de perles vertes et se trouve dans une solitude atroce. Il est obligé d'affronter les conditions

\footnotetext{
${ }^{19}$ Ibid., p. 209.

${ }^{20}$ Ibid., Loc.cit.

${ }^{21}$ Ibid., p.p. 209, 210.

${ }^{22}$ Ibid., p. 211.

${ }^{23}$ Ibid., p. 99.
} 
les plus macabres. Entassé avec d'autres migrants dans une camionnette, les passeurs les déchargent pour les racketter. Envahi par la rage, Soleiman tend de s'y opposer. Il reçoit en retour des coups et sera laissé par terre comme mort. A son réveil, il ressent la douleur. Les passeurs lui ont tout volé. Tout le monde est parti sauf Boubakar, un petit homme maigre qui boite, d'où le nom du chapitre «le boiteux ». Face à ce monde hostile, la rencontre avec Boubakar, s'avère providentielle. C'est cette rencontre qui lui rend ce qui fait de lui un homme : la solidarité humaine.

$\mathrm{Au}$ niveau spatial, les deux trajets initiatiques mènent donc les personnages principaux dans des directions opposées, du nord au sud et du sud au nord. Alors que Soleiman part de l'Afrique vers l'Europe, Salvatore, lui, part de l'Europe vers l'Afrique.

Cependant, et par une structure très savamment élaborée, les deux trajets se rencontrent à Ghardaïa, sorte de passage obligé de tous les migrants. En fait, la rencontre des deux protagonistes marque une étape ultime dans leur vie. Elle achève leurs transformations. Leurs trajets se rencontrent une fois pour toute pour diverger définitivement. Les deux personnages ne font qu'un au moment où leurs destinées se rencontrent à Ghardaïa. Après cette rencontre, Soleiman atteint son but alors que Salvatore meurt.

A Ghardaïa, triste et déçu, Piracci découvre qu'il n'avait aucune chance à trouver son eldorado car il n'en avait point. En même temps et toujours à Ghardaïa, Soleiman atteint le comble du désespoir et du dégoût après avoir assommé et volé un marchand algérien. C'est en ce moment qu'il rencontre Piracci sur la place et qu'il le prend pour l'ombre de Massambalo. Il lui offre le collier de perles vertes que lui avait donné son frère. Après réflexion, Piracci décide de jouer le jeu. Il accepte l'offrande pour donner au pauvre migrant qu'est Soleiman la force de l'espoir et de la foi. Salvatore est encore une fois sauveur en arrachant Soleiman au désespoir.

Voici que Piracci a trouvé son but. «Seul comptait pour lui qu'il avait trouvé ce qu'il ferait désormais. [...], il ne serait plus qu'une ombre qui donne courage aux hommes. [...] Il n'y avait qu'ainsi qu'il pouvaient encore appartenir au monde. ${ }^{24}$ Salvatore trouve enfin un sens à sa vie. «Face à ce jeune homme, il comprenait que l'eldorado existait pour les autres et

${ }^{24}$ Ibid., p. p. 234, 235. 
qu'il était en son pouvoir de faire de sorte qu'ils ne doutent pas de leur chance. [...] Un calme profond l'habitait. ${ }^{25} \mathrm{C}$ 'est ainsi qu'il atteint son eldorado. Il trouve ainsi un but à sa vie et atteint la tranquillité qu'il cherchait. Cependant, bouleversé par un de ces camions qui transportent les migrants, il meurt.

En mourant, Piracci s'imagine parler aux clandestins qui sont sur la route de l'Europe. Il fait tout à fait le contraire de ce qu'il faisait au début du roman. Le commandant qui interceptait les migrants au début du roman, les encourage à la fin du roman et de sa vie.

«Il parlait à la terre et aux peuples en souffrance. [...] Il voulait que sa voix coure le long des routes et des sentiers. L'Eldorado était là. Il ne fallait pas tarder. [...] Il leur dit de partir, sans attendre, à l'assaut des frontières. De tenter leur chance avec rage et obstination. Que des terres lointaines les attendaient. [...] Que l'Eldorado était là. Et qu'il n'était pas de mer que l'homme ne puisse traverser. ${ }^{26}$

Le voyage initiatique de Piracci l'a mené vers sa mort. On dirait que dès le début du roman cette fin se préparait. Dès le premier chapitre, celui qui ouvre le récit de Piracci, la mort est omniprésente avec les « centaines de poissons morts» au marché telle une «macabre exposition». Le mot «mort» est utilisé vingt-deux fois dans ce chapitre. Piracci se rend compte que la femme du Vittoria est venue le voir pour lui dire «qu'il était temps de [s]e mettre en route pour trouver [s] a mort. ${ }^{27}$

En acceptant de devenir pour Soleiman l'ombre de Massambalo, il se condamne lui-même à devenir une ombre et finalement à disparaître car «[...] : les ombres du dieu des émigrés ne peuvent être vues qu'une fois, après quoi elles s'évanouissent. $\gg{ }^{28}$ Comme toutes les ombres, il disparait.

Or, cette rencontre décisive entre Piracci et Soleiman est le seul endroit où les deux récits se croisent. En fait, aucun indice temporel ne permet de situer le déroulement des deux récits. Ce n'est qu'au chapitre X, qu'on

\footnotetext{
${ }^{25}$ Ibid., p. 232.

${ }^{26}$ Ibid., p. 237.

${ }^{27}$ Ibid., p. 212.

${ }^{28}$ Ibid., p. 237.
} 
apprend que Soleiman a quitté les siens depuis huit mois. Toutefois, la structure du roman qui fait que les deux récits avancent simultanément laisse suggérer que les voyages de Soleiman et de Piracci ont lieu simultanément aussi. Cependant, un certain décalage temporel intervient progressivement pour que la rencontre à Ghardaïa ait lieu plus tôt dans le récit du Soleiman que dans celui de Piracci.

En effet, cette rencontre est racontée différemment selon la focalisation adoptée. Ainsi, au chapitre VIII, voire vers le milieu du roman, Soleiman nous apprend qu'à Ghardaïa, il rencontre quelqu'un qu'il reconnaît et sans que le lecteur ne comprenne de qui il s'agit, il fait «ce qu'il doit» et lui offre le collier de Jamal. Cette rencontre, mystérieuse transforme Soleiman. Il n'a plus peur. Ce n'est qu'au dernier chapitre du roman qui porte justement le titre de «L'ombre de Massambalo » qu'on a le point de vue de Salvatore qui explique et élucide la situation. On comprend que c'est Piracci que Soleiman a rencontré et qu'il a pris pour l'ombre de Massambalo. Ce procédé narratif permet effectivement de garder le suspense jusqu'aux dernières pages du roman.

L'autre transformation vécue par Soleiman au cours de son voyage initiatique est bien sa relation avec Boubakar. Au moment de leur passage des fils barbelés à Ceuta, ces fils qui séparent le sol espagnol du sol marocain, Soleiman, plus rapide que Boubakar, a dû retourner pour le sauver. Si son voyage l'a effectivement mené à son eldorado, il n'y est arrivé que parce qu'il a découvert la solidarité humaine. S'il avait laissé Boubakar derrière lui et couru comme une bête, il serait passé car il est plus rapide que son compagnon boiteux, mais il serait damné. "Soleiman serait devenue une bête laide qui piétine ses frères. C'est pour cela, sûrement, que je suis allé chercher Boubakar et que je l'ai aidé. Pas pour le sauver lui mais pour me sauver moi. Si je l'avais laissé accroché aux barbelés, je n'aurais plus jamais trouvé le sommeil et j'aurais foulé ces terres nouvelles sans un frisson de plaisir. » ${ }^{29}$

$\mathrm{Au}$ cours de l'initiation de Piracci et de Soleiman, paradoxalement, alors que la condition matérielle de Salvatore s'est dégradée, il devient pauvre et maigre, il s'est libéré et a trouvé sa raison de vivre. Il décide de passer pour l'ombre de Massambalo qui protège les migrants, leur donne

${ }^{29}$ Ibid., p. 224. 
espoir et force. Quant à Soleiman, une fois arrivé, il saigne, son corps est assailli de douleur, ses os sont fracturés, ces plaies sont ouvertes mais il sent la terre nouvelle sous lui et goûte la joie de la libération en entrant à Ceuta.

En fait, Salvatore n'a pas toutes les caractéristiques du héros. On pourrait même dire qu'il se rapprocherait davantage de l'antihéros. Alors que Soleiman et Boubakar sont en revanche plus proches de la figure du héros proprement dit. Bien qu'au début, Salvatore incarne l'autorité, après la remise en question de sa vie, il incarne les valeurs de la bonté, de la solidarité humaine et de la fraternité. Sa compassion envers les migrants le pousse à découvrir leur vie. Malheureux, il ne parvient pas à donner un sens à sa vie. Sauvé du suicide, il meurt dans l'anonymat et l'indifférence renversé par un camion.

Soleiman, lui, est plus positif. Il est ainsi plus proche de la figure du héros. Optimiste et enthousiaste, il réussit son voyage et arrive à réaliser son rêve. Cependant, comme tout être humain, il a ses imperfections. A un moment de désespoir, il assomme le marchant algérien pour le voler. Il regrette ensuite son acte et décide de ne plus se comporter comme une bête. Son comportement héroïque en revenant pour sauver son ami Boubakar et l'aider à franchir la frontière espagnole, est l'acte qui, en faisant de lui un vrai héros, le sauve et le libère.

Les héros ou anti-héros d'Eldorado emmènent le lecteur avec eux dans le tumulte de leur voyage. D'ailleurs, la notion de voyage est une notion-clé chez Gaudé. Au cours de son entretien dans l'émission Hep taxi! Gaudé se définit comme un "Homme de voyage » car, pour lui, le voyage est «la seule expérience lors de laquelle on peut se permettre de n'être personne » 30. Or, le voyage qu'il choisit d'aborder dans son roman, celui des migrants, s'avère l'un des plus macabres et des plus tristes. Tel qu'il le formule si bien, c'est un voyage qui "impose ses épreuves et nous vieillissons à chacune d'entre elles.» ${ }^{31}$ Mais c'est un vrai voyage initiatique qui nous entraine, malgré toutes les difficultés, vers l'Eldorado.

\footnotetext{
30 «Laurent Gaudé dans le taxi de Jérôme Colin : L'interview intégrale », Interview de Laurent Gaudé dans l'émission Hep Taxi!, RTBF, $1^{\mathrm{er}}$ mars 2015, https://ds1.static.rtbf.be/article/pdf/gaude-1425045511.pdf, consulté 11 mars 2018.

31 Eldorado, p. 159.
} 
En mettant la lumière sur un des problèmes les plus brûlants de la région, Eldorado apporte surtout une vision humaine sur la notion de voyage, choisi ou subi. En effet, Gaudé affirme qu'il établit

«une différence entre les réfugiés qui doivent partir, comme, aujourd'hui, notamment, les habitants d'Afghanistan, d'Érythrée et de Syrie, et les migrants qui ont choisi de partir, comme, par exemple, les Maliens ou les Sénégalais. Il est évident que les réfugiés syriens n'ont pas de désir dans les yeux, mais de l'épuisement. Ils sont cassés et je respecte cet état qui laisse sans voix. Mais il y a des émigrés qui rêvent d'une vie meilleure et je les respecte aussi pour cet élan-là. » ${ }^{32}$

Dans le roman, les passages qui décrivent le voyage des migrants sont nombreux. Au début, c'est la femme du Vittoria qui décrit à Salvatore son aventure tragique. Plus loin, dans le récit de Soleiman, certains passages sont très mouvementés comme dans le chapitre de l'assaut (chapitre X) ou de l'entrée en Espagne (Chapitre XII). Le lecteur vit avec les personnages leur traversée des frontières.

Lorsque Soleiman et Boubakar mettent les pieds sur le sol européen en franchissant les frontières entre l'Espagne et le Maroc, Gaudé ne manque pas de rappeler ainsi cette anomalie des frontières. Rappelons qu'en août 2005, un an avant la publication d'Eldorado, de nombreux migrants subsahariens avaient essayé de franchir les grillages barbelés de Ceuta et Melilla, les deux enclaves espagnoles dans le Nord du Maroc, avec pour conséquences de nombreux décès. C'est justement ce que décrit Gaudé avec beaucoup de réalisme.

Le choix de l'emplacement spatial est très significatif car, en fait, Ceuta et Melilla sont aujourd'hui au cœur du problème migratoire. Ces deux villes sont effectivement depuis la fin $\mathrm{du} \mathrm{XV}^{\mathrm{e}}$ siècle des territoires espagnols. En effet, si un migrant arrive à mettre le pied dans ces villes, il

\footnotetext{
32 «Laurent Gaudé : Je suis attiré par les sujets qui portent du tragique », Marie-Pierre GENECAND, 27 septembre 2015, https://www.letemps.ch/culture/laurent-gaude-suis-attire-sujets-portent-tragique, consulté le 15 mars 2018.
} 
est sur le sol européen bien qu'il soit encore sur le continent africain et la traversée des fils barbelés s'avèrent moins risqué que la traversée en mer.

Dans le roman, Soleiman ne manque pas de relever avec étonnement et ironie, qu'une fois en Espagne, les soldats espagnols vont et viennent pour distribuer aux clandestins de l'eau et des soins. Ces mêmes soldats qui les battaient quelques minutes auparavant pour les faire reculer, une fois passés, veillaient sur eux avec calme et attention.

"Qu'est-ce qui les empêche de se ruer sur nous et de continuer ce qu'ils ont commencé? [...] Ce sont les mêmes bras qui tiennent ces tasses en plastique et qui frappaient sur nos têtes. Les mêmes yeux qui nous traquent quelques instants plus tôt et semblent maintenant ne plus nous voir. [...] Nous sommes passés. C'est un jeu et nous avons gagné. Ils respectent les règles. ${ }^{33}$

Le roman met en outre la lumière sur le scandale du trafic des clandestins et les réseaux de passeurs. Arrivé en Lybie, Salvatore est arrêté par la police. Cependant, un policier corrompu le conduit chez la Reine d'Al-Zuwarah, une horrible femme riche et obèse à la tête d'un réseau de passeurs. En effet, les passeurs demandent des sommes exorbitantes aux migrants pour les transporter en Europe. Ils les rackettent pour la plupart du temps comme il est arrivé à Soleiman ou ils les laissent en plein mer comme c'est le cas de la femme du Vittoria.

Malgré l'inspiration d'un sujet qui brûle d'actualité, la veine mythologique n'est point absente d'Eldorado. Le voyage de Salvatore, seul, sur une barque en Méditerranée rappelle bien les périples d'Ulysse dans l'Odyssée. En outre, l'apparition de Boubakar au chapitre VI, intitulé justement «Le boiteux », marque l'arrivée presque miraculeuse d'un grand soutien pour Soleiman. Il symbolise l'aide providentielle, quasi divine pour soutenir un homme arrivé au fond de sa détresse.

$\mathrm{Au}$ niveau symbolique, boiter est le contraire de marcher droit. Le boiteux n'a évidemment pas l'équilibre normal. En effet, Boubakar est en route depuis huit ans pour pouvoir rejoindre de façon illégale l'Europe. D'autre part, les mythes antiques mettent en scène des héros et des dieux

${ }^{33}$ Ibid., p. 223. 
boiteux. Dionysos, Héphaïstos et Harpocrate boitent. D'autre part, Héphaïstos est le dieu du feu et des forgerons. Or, l'enfer est bien ce par quoi sont passées Boubakar et Soleiman au cours de leur voyage. Ils sont effectivement tel que le dit le titre du chapitre XII, des « Frères de l'enfer.» ${ }^{34}$

On ne peut manquer de rappeler également Sisyphe. En fait, lorsque Piracci menait une vie simple et aisée, il ne trouvait aucun sens à sa vie. Il entreprend un voyage dangereux à la recherche de son Eldorado. Et s'il ne le trouve point, il comprend que l'effort lui-même déployé pour y arriver est capable de remplir son âme, tout comme Sisyphe condamné à refaire toujours le même acte mais pour qui comprend que la lutte elle-même donne un sens à la vie.

En conclusion, dans ce monde de plus en plus triste, Eldorado de Laurent Gaudé vient nous rappeler la beauté de la littérature. Son vrai apport est de donner un visage humain à la question de l'immigration clandestine. Contrairement aux émissions de télévision et aux rapports de presse, chez lui, les êtres humains ne sont point des chiffres, des statistiques, des graphiques, des noyés et des morts. Ces visages que nous voyons à la télévision ou dans les journaux ont dans le roman de Gaudé une identité, un nom et une histoire. Ce sont des personnages qui ont leurs rêves et leurs espoirs et ils acquièrent ainsi toute leur dignité humaine, voire leur beauté.

Le roman de Gaudé, en effet, diffère de tout autre roman faisant partie de la littérature migrante. Ces romans, écrits pour la plupart par des migrants francophones, sont les récits de voyage de migrants en route vers l'Europe. Écrit par un français, Eldorado a le privilège de présenter à la fois le point de vue européen et le point de vue opposé, celui des migrants. C'est d'ailleurs justement ce qui a marqué la critique tel que le remarque Philippe Chevilley : "Eldorado raconte l'odyssée de ces déçus de la terre promise, anéantis par des passeurs criminels... et des européens convaincus qu'ils ne peuvent accueillir toute la misère du monde et qui ferment les yeux pour ne plus savoir ce qui se joue et se meurt à leur porte. ${ }^{35}$

\footnotetext{
${ }^{34}$ Ibid., p. 217.

${ }^{35}$ CHEVILLY, Philippe, «Le drame des clandestins », in Les Échos, le 12 septembre 2006, https://www.lesechos.fr/12/09/2006/LesEchos/19749-052-ECH_le-drame-des-clandestins.htm, consulté le 3 mars 2018.
} 
Laurent Gaudé est également flasciné par la notion de désir, ce désir qui pousse les migrants à entreprendre des voyages coûteux, macabres et dangereux à la poursuite de leurs rêves et à la recherche de leur eldorado. L'eldorado mythique et chimérique devient, pour eux, une réalité à laquelle ils ne renonceront pas quitte à mourir.

«Je suis bouleversé et fasciné de voir que parfois ces hommes mettent des années, sept, huit ans pour n'atteindre que le bord de la Méditerranée. Il faut un désir forcené, une obstination hors du commun. Mais c'est aussi un fantasme très européen de se dire que les pauvres ont en eux une soif énorme et un désir de tout, et que la vieille Europe se meurt... Disons que confrontés à eux, nous ressentons à quel point leur désir est autrement plus cru, plus violent, plus puissant, que celui que le nôtre. A nous de réinventer le désir dans nos vies. C'est la chose la plus précieuse que nous ayons en nous. $»^{36}$

Le roman de Gaudé s'avère effectivement d'une grande richesse aussi bien sur le plan thématique que sur le plan strictement littéraire. Tel que nous venons de le voir, la structure binaire des chapitres et les personnages, venant de part et d'autre de la Méditerranée permettent de poser différents regards sur la question de l'immigration. Sans proposer de solutions ou donner de jugements, Eldorado nous invite à réfléchir, à considérer la question d'un autre point de vue et c'est là où réside toute sa richesse, son apport et sa particularité.

A travers la narratologie savamment élaborée, le lecteur se trouve à la place de ces migrants. Il parvient à partager leurs émotions, leurs tristesses et leurs joies. Ce lecteur, surtout occidental, se trouve confronté à un autre point de vue. Le désir, absurde et illogique, des migrants de rejoindre le continent européen acquiert soudain un autre sens. Il devient, sous la plume

\footnotetext{
${ }^{36}$ « Entretien. Laurent Gaudé. Eldorado ou la chimère qui nous fait croire », Isabelle FALCONNIER, Art.cit.
} 
de Gaudé, l'incarnation même de l'espoir et de la volonté. Ainsi, devient-il non seulement légitime mais aussi respectable.

Laurent Gaudé parvient à nous faire sentir la beauté de l'espérance, la volonté et la détermination, la force du rêve et de la foi. A travers sa narration, il donne la voix à tous ceux qui ne peuvent pas se laisser entendre, tous ceux qui n'ont pas de voix, mais pour qui la voie est claire et déterminée. Ces migrants anonymes acquièrent enfin dans le roman, comme dans nos esprits, toute leur humanité.

Le roman excelle à mettre en lumière la lutte que doit mener chacun au cours de sa vie : lutte pour arriver à une vie meilleure, lutte pour réaliser ses rêves et tenir ses promesses, lutte contre la pauvreté, mais lutte aussi contre soi-même, contre ses instincts, lutte contre la maladie, lutte aussi contre la monotonie d'une vie sans but et sans sens. Et malgré toutes les tristesses, le lecteur retrouve les lueurs d'espoir. La fraternité de Soleiman et Boubakar est exceptionnelle et grâce à leur solidarité ils réalisent leur rêve, posent ensemble le pied sur le sol européen et atteignent leur eldorado.

Il va sans dire qu'Eldorado a reçu une réception très favorable de la part de la critique, tant en France qu'à l'étranger. Vu son importance et l'importance du sujet qu'il traite, le roman a été même intégré dans les classes au niveau scolaire ${ }^{37}$.

Peut-on dire que Laurent Gaudé est un écrivain engagé ? Il l'affirme lui-même:

«Je suis convaincu qu'un livre engagé peut changer le monde et la face de l'histoire. Je veux partager avec mes lecteurs le regard que j'ai posé sur ces hommes partis du fond de l'Afrique, sur ceux qui partent, de manière générale, ceux qui ont eu envie de tout quitter, que je trouve courageux. On peut parler d'eux autrement que de la manière dont en parlent les discours politiques. C'est effectivement un problème politique, mais mon travail consiste à montrer que l'on peut en parler aussi de manière littéraire, humaniste. J'espère pouvoir

\footnotetext{
${ }^{37}$ C'est ce qui explique les deux profils littéraires concernant ce roman : Camille FRAIPONT, Eldorado de Laurent Gaudé, Analyse d'œuvre, Profil Littéraire, 2015 et Agnès FLEURY et René HENRI, Eldorado de Laurent Gaudé, Analyse de l'œuvre, Le PetitLittéarire.fr, 2014.
} 
changer un peu le regard que les lecteurs, et même les politiques, vont porter sur cette réalité. $»^{38}$

A travers son écriture, Gaudé se place à l'encontre du discours politique, celui qui considère l'immigration comme étant une guerre contre l'Europe, conception que Piracci a d'ailleurs évoqué lorsqu'il s'est décrit comme le gardien de la forteresse Europe. Gaudé refuse de "criminaliser » ceux qui ont choisi d'immigrer. Si les discours politiques parlent d'eux comme étant «des clandestins, des illégaux, des sans-papiers», lui, il cherche à dire «qu'ils sont courageux, forts, beaux dans leur désir. ${ }^{39} \mathrm{Il}$ invite les lecteurs, comme les politiciens, à jeter un autre regard pour pouvoir concevoir autrement la question.

Après l'écriture d'Eldorado, le sujet n'a plus quitté son auteur. Gaudé cherchait à l'investir... Ce ne pouvait pas être juste un sujet de livre, un dossier qu'on referme. Il fallait que tout ce travail, cette sensibilisation serve à quelque chose. C'est lui donc qui a fait la démarche d'entrer en contact avec Amnesty International en leur envoyant un mail affirmant qu'il était disponible surtout pour le problème des réfugiés. "Il fallait que JE serve à quelque chose, que je continue à accompagner cette problématique. ${ }^{40}$

Dans le cadre du concours «Vos talents pour les réfugiés », Laurent Gaudé rejoint le jury national de ce concours créatif qui a pour objectif de promouvoir une France accueillante et solidaire. Il rêve d'inventer une hospitalité européenne. Il rêve d'une Europe qui puisse accueillir ces milliers d'hommes, de femmes et d'enfants qui fuient la guerre, la peur, la barbarie et ne rêvent que de paix. Comme eux, ne sommes-nous pas tous, à un moment de notre vie, des émigrés à la recherche de la paix ?

\footnotetext{
38 « Entretien. Laurent Gaudé. Eldorado ou la chimère qui nous fait croire », Isabelle FALCONNIER, Art.cit.

${ }^{39}$ Ibid., Loc.cit.

40 «Entretien avec Laurent Gaudé, écrivain engagé », Virginie PLAUT, Art.cit.
} 
Amany GHANDER Département de langue et de littérature françaises

Faculté des Lettres Université d'Alexandrie 